\title{
Female Adolescent Soccer Players Utilize Different Neuromuscular Strategies Between Limbs During the Propulsion Phase of a Lateral Vertical Jump
}

\author{
Matthew D DeLang, PT, DPT, CSCS $^{1}$, Joseph P Hannon², Shiho Goto ${ }^{2}$, James M Bothwell², J Craig Garrison ${ }^{3}$ \\ ${ }^{1}$ Right to Dream Academy, ${ }^{2}$ Texas Health Sports Medicine, ${ }^{3}$ Northwestern University \\ Keywords: between-limb, vertical jump, limb dominance \\ https://doi.org/10.26603/001c.22134
}

\section{International Journal of Sports Physical Therapy}

Vol. 16, Issue 3, 2021

\section{Background}

Multiplanar dynamic stability is an important unilateral function in soccer performance but has been scarcely examined in female soccer players. The lateral vertical jump task assesses unilateral functional performance, and energy generation contribution examines how each joint (hip, knee, ankle) contributes to the vertical component of the vertical jump phase to measure inter- and intra-limb differences.

\section{Purpose}

To examine dominant versus non-dominant limb performance using energy generation contribution of the hip, knee, and ankle during the vertical jump component of the lateral vertical jump.

\section{Study Design}

Cross-sectional observational study.

\section{Methods}

Seventeen healthy, adolescent female soccer players (age $13.4 \pm 1.7$ years; height $160.6 \pm 6.0$ $\mathrm{cm}$; mass $53.1 \pm 8.2 \mathrm{~kg}$ ) participated. Quadriceps strength was measured via isokinetic dynamometry. Energy generation contribution (measured from maximal knee flexion to toe off) and vertical jump height were measured during the vertical component of the lateral vertical jump.

\section{Results}

There was no significant difference between limbs for quadriceps strength $(p=0.64)$, jump height $(p=0.59)$, or ankle energy generation contribution $(p=0.38)$. Energy generation contribution was significantly greater in the dominant hip (dominant $29.7 \pm 8.6 \%$, non-dominant $18.4 \pm 6.3 \%, \mathrm{p}<0.001$ ) and non-dominant knee (dominant $22.8 \pm 6.8 \%$, non-dominant $36.2 \pm 8.5 \%$, $\mathrm{p}<0.001)$.

\section{Conclusion}

High demand on coordination and motor control during the lateral vertical jump and inherent limb dominance may explain different intra-limb strategies for task performance despite jump height symmetry. Non-dominant affinity for stability and dominant compensatory performance may neutralize potential asymmetries. Implications for symmetry in observable outcomes such as jump height must consider underlying internal asymmetries.

\footnotetext{
a Corresponding Author: 


\section{Levels of Evidence}

3B

\section{Clinical Relevance}

Symmetrical findings on functional tasks have underlying internal asymmetries observed here in female adolescent soccer players. The lateral vertical jump may highlight these internal asymmetries (hip- versus knee-dominant movement strategies) due to the high coordinative demand to perform the task. Clinicians should be cognizant of underlying, potentially inherent, asymmetries even when observing functional symmetry in a task.

\section{What is known about the subject}

Female adolescent soccer players are a high-risk cohort for sustaining anterior cruciate ligament injuries. Limb dominance may play a role in the performance of functional tasks, and limb dominance in soccer players is quite specialized: the dominant limb is the preferred kicking limb, while the non-dominant limb is the preferred stabilizing limb (plant leg). Functional performance in female soccer players has been studied in kicking, dribbling, sprinting, change of direction, and jumping - however, these tasks were measured independent of limb dominance. It remains to be seen how unilateral functional tasks may be affected by limb dominance in female adolescent soccer players.

\section{What this study adds to existing knowledge}

This study provides data on functional performance relative to limb dominance in female adolescent soccer players, and captures the lateral vertical jump task in both inter- and intra-limb measures. This highlights that intra-limb strategies to perform a coordinated motor task may be different between limbs, herein attributed to limb dominance. Even if gross motor outputs between limbs are symmetrical (i.e. jump height), the underlying movement strategies to achieve that output may be different (hip- versus knee-dominant movement strategies). These findings are important to research on functional performance measures related to attaining between-limb symmetry, as measures of energy generation contribution open the door for a more thorough understanding of joint-by-joint intra-limb contributions during a functional task.

\section{INTRODUCTION}

Female soccer has grown to include over 30 million worldwide participants. ${ }^{1}$ Between 2013 and 2017, youth female soccer participation has increased by $70 \% .^{2}$ This impressive growth has not been equaled by corresponding scientific literature, where a dearth of research exists in the women's game, especially when compared to their male counterparts. $^{3}$

Soccer has a large focus on tactics and strategy. ${ }^{4}$ On-field tactical decisions regularly come down to player positioning and the capabilities of those players, often with consideration for limb dominance. Limb dominance specific to soccer is often dichotomized into a dominant kicking limb and a non-dominant support limb, ${ }^{5}$ which is determined by asking the question: "which is your preferred foot to kick the ball?"6 In sport-specific functional tasks, the dominant limb in male soccer players produces greater ball velocity during maximal instep kicks, 7,8 performs quicker changes of direction, ${ }^{5}$ and has better intersegmental patterning than the non-dominant limb. ${ }^{9}$ In contrast, limb dominance comparisons relative to functional testing in female soccer players have been scarcely reported. Limb dominance during functional testing has previously been reported in a group of healthy female soccer players, with no significant differences between dominant and non-dominant limb for the drop vertical jump in knee motion or knee abduction moments. ${ }^{10}$ In a sample of first-team Swedish female players,
Östenberg et al. ${ }^{11}$ reported no between-limb differences in a series of functional jump and hop test distances when comparing right versus left or the dominant versus nondominant limbs.

Functional testing methods in athletes should ideally replicate the demands of a particular sport. ${ }^{12}$ Soccer is embodied by multidirectional and multiplanar movements with variable locomotor demands involving running backwards and sideways ${ }^{13}$ and a change in locomotor activity every four seconds in elite women's competition. ${ }^{14}$ Previous research examining functional testing in female soccer players includes studies of kicking and dribbling, ${ }^{15}$ sprinting, ${ }^{15-19}$ changing directions, ${ }^{17}$ and an array of jumping tasks, ${ }^{15-17,19-21}$ all of which were assessed independent of limb dominance. The jumping tasks reported in the literature are often unidirectional and uniplanar (i.e. vertical jump, single leg hop, triple hop, drop jump, broad jump), although a recent study by Bishop et al. ${ }^{20}$ suggests the multiplanar nature of soccer warrants multiple jump tests to be used to classify interlimb asymmetries. The lateral vertical jump task is a performance measure that can be used to assess multiplanar dynamic stability. It has previously been studied at time of return to sport in female athletes following anterior cruciate ligament (ACL) reconstruction and compared to age- and activity-matched healthy female controls. ${ }^{22}$ Females in the ACL reconstruction group utilized a greater energy absorption contribution (measure of contributions of the hip, knee, and ankle during the landing phase of the lateral vertical jump) through both the in- 
volved and uninvolved hips than females in the healthy group. While these findings demonstrate altered energy absorption strategies utilized during the lateral vertical jump, the focus was on how the individual loaded the limb during the landing portion of the task. This study rather will focus on the energy generation performed during the propulsion phase of the lateral vertical jump. As such, the aim of the current study was to examine dominant versus non-dominant limb performance using energy generation contribution of the hip, knee, and ankle during the vertical jump component of the lateral vertical jump. With side-to-side differences demonstrated in single leg countermovement jump in a similar population, ${ }^{20}$ we hypothesized that female adolescent soccer players will produce greater total energy generation, demonstrated by vertical jump height, in the dominant limb than the non-dominant limb. We also hypothesized that the dominant limb would demonstrate greater energy generation contribution in the hip and knee and similar energy generation contribution in the ankle when compared to the non-dominant limb.

\section{MATERIALS AND METHODS}

This study investigated the differences between dominant and non-dominant limb performance using a novel lateral hop to vertical jump measure of dynamic stability. A crosssectional design was implemented following the STROBE (Strengthening the Reporting of Observational studies in Epidemiology) statement. ${ }^{23}$ The dominant limb was determined by self-report, defined as the limb that the participant reported she would choose to kick a ball. The lateral vertical jump task was divided into energy generation contributions of the hip, knee, and ankle joints to assess if within-limb strategies differed between the dominant and non-dominant limb.

\section{PARTICIPANTS}

A total of 17 healthy female soccer players (Table 1) volunteered to participate in this study. These adolescent soccer players (age $13.4 \pm 1.7$ years) were a subset of a larger cohort study with inclusion criteria: between the ages of 13 and 25 years and involved in a level 1 sport (involving cutting, planting, pivoting, jumping, and landing) for at least 50 hours/year. A power analysis was calculated to estimate sample size using energy generation contribution as the variable of interest and it was determined that seventeen participants were needed to detect statistical significance $(80 \%$ power at $\mathrm{p}<0.05)$. Participants were considered for study inclusion if they scored $\geqslant 95 \%$ on the International Knee Documentation Committee (IKDC) subjective form and did not experience any lower extremity orthopedic injuries within 3 months before testing. After the screening process, eligible participants were invited to take part in the study. Since participants were minors, parental permission and child assent were obtained and the rights of each person were protected. The institutional review board of Texas Health Resources approved all research procedures. After enrollment in the study, information regarding injury history and athletic involvement was collected from each participant. Height and body mass were measured for each
Table 1: Participant demographics $(n=17)$.

\begin{tabular}{l|c}
\hline Age (years) & $13.4 \pm 1.7$ \\
\hline Height (cm) & $160.6 \pm 6.0$ \\
\hline Mass (kg) & $53.1 \pm 8.2$ \\
\hline Soccer Participation (hours/week) & $7.4 \pm 3.6$ \\
\hline Season Duration (months) & $10.8 \pm 2.3$ \\
\hline IKDC Score & $99.5 \pm 1.3$ \\
\hline
\end{tabular}

IKDC= International Knee Documentation Committee

participant using an eye-level weigh beam scale (Detecto, Webb City, MO, USA).

\section{PROCEDURES}

\section{3-DIMENSIONAL MOTION ANALYSIS}

Retroreflective marker placement was consistent with a previously published protocol ${ }^{22}$ and attached to the participant with double-sided tape. Marker placement included the spinous process of the seventh cervical vertebra, twelfth thoracic vertebra, between the fourth and fifth lumbar vertebrae, sternum, bilateral acromion process, anterior superior iliac spine, posterior superior iliac spine, greater trochanter, anterior thigh, medial and lateral epicondyles of the femur, anterior shank, medial and lateral malleoli, calcaneus, and first and fifth metatarsal heads. Three additional retroreflective markers were attached on the sacrum as a cluster. Qualisys motion capture system with 10 Oqus cameras (Qualisys, Göteborg, Sweden) were used to collect three-dimensional coordinates of reflected markers attached to the participant at a sampling rate of $120 \mathrm{~Hz}$. Two force plates (AMTI, Watertown, MA, USA) were used to collected ground-reaction forces at a sampling rate of $1200 \mathrm{~Hz}$, and kinematic and force plate data were time synchronized. After completion of the static trial, markers of the medial femoral epicondyle, medial malleolus, and bilateral anterior superior iliac spine were removed, and participants performed the lateral vertical jump.

\section{LATERAL VERTICAL JUMP}

Participants began this unilateral jump task by standing to the side of the force plate at a distance equal to $50 \%$ of their height with the testing limb closest to the force plate. The dominant limb was tested first. Fifty percent of the participant's height was used to ensure that participants are challenged with the task while also normalizing for individual height differences. ${ }^{22}$ Participants were asked to hop onto the force plate by pushing off the ground with their nontesting limb, land with the testing limb on the force plate, and then immediately perform a maximal single leg vertical jump and land back onto the force plate with their testing limb (Figure 1). Immediately after landing on the force plate, the participants were required to stabilize themselves on the force plate for approximately two seconds. Unilateral vertical jump height was captured in centimeters extrapolated from flight time. Participants warmed up on an exer- 

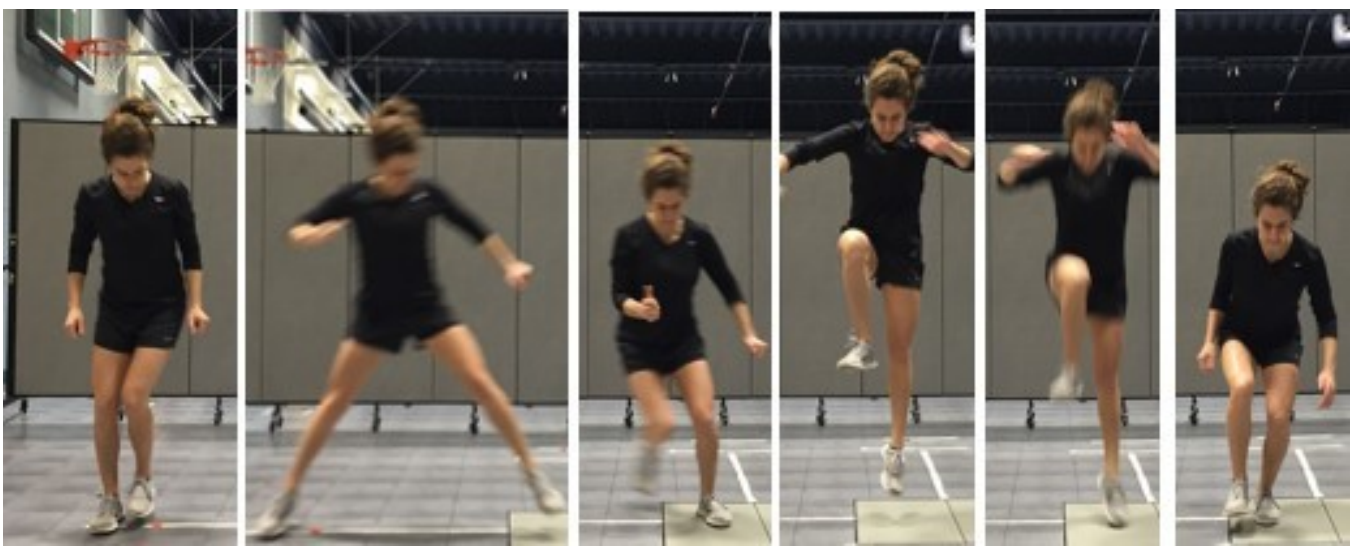

Figure 1: The lateral vertical jump

cise bike before performing three trials on each limb. Trials were excluded and repeated if the participant's entire foot did not contact the force plate, if the non-testing limb contacted the ground or force plate upon landing or immediately after, or if the participant did not maintain balance.

\section{DATA PROCESSING AND REDUCTION}

Three-dimensional joint coordinates were estimated from the trajectories of the reflective markers. All kinematic and kinetic data were exported into Visual3D software (C-Motion) to process and reduce data. The kinematic and force data were filtered via a fourth-order low-pass Butterworth filter with a zerophase lag at $10 \mathrm{~Hz}$. The lower extremity joint angles were calculated using an inverse kinematic approach, and the lower extremity internal moment was calculated using an inverse dynamic approach. The energy generation contribution was calculated by integrating the positive part of the power curve where joint angular velocity and net joint moment are in opposite directions, indicating concentric loading during the vertical jump takeoff phase from maximal knee flexion to toe-off. The energy generation contribution for each joint was normalized to the product of height and weight and averaged across three trials.

\section{ISOKINETIC DYNAMOMETRY}

The Multi-Joint Testing and Rehabilitation System (Biodex Medical Systems) was used for testing isokinetic quadriceps muscle strength. Participants were seated on the Biodex system and secured with padded straps around the thigh, pelvis, and torso to minimize accessory and compensatory movements during testing. The femoral condyle of the testing limb was aligned with the dynamometer axis of rotation following the manufacturer's instructions. A warmup consisting of five submaximal knee extension/flexion contractions (60 degrees/second) was provided to familiarize each participant with the testing motion. Next participants performed five maximum effort trials. The average of the five trials was normalized to body mass $(\mathrm{Nm} / \mathrm{kg})$ and used for data analysis.

\section{STATISTICAL ANALYSES}

All data analyses were performed using SPSS version 23 (International Business Machines Corporation, Armonk, NY). Multiple paired t-tests were used to assess between-limb differences in energy generation contribution at the hip, knee, and ankle and quadriceps strength. A Bonferroni correction was applied to account for multiple comparisons and the alpha level was set at $\mathrm{P}<.01$. Effect sizes were estimated for each variable of interest using Cohen's $d$ formula.

\section{RESULTS}

Table 2 demonstrates the between limb differences for outcome variables including vertical jump height, quadriceps strength, and energy generation contribution at the hip, knee, and ankle. The dominant limb demonstrated significantly greater hip energy generation contribution (29.7 $\pm 8.6 \%)$ than the non-dominant limb $(18.4 \pm 6.3 \%, \mathrm{p}<$ $0.001)$. At the knee, the dominant limb produced significantly less energy generation contribution than the nondominant limb (dominant 22.8 $\pm 6.8 \%$, non-dominant $36.2 \pm 8.5 \%, p<0.001)$. There were no other significant differences between the dominant and non-dominant limbs for ankle energy generation contribution $(p=0.38)$, jump height $(p=0.59)$, or quadriceps strength $(p=0.64)$. Effect sizes using Cohen's $d$ are also reported in Table 2.

\section{DISCUSSION}

This study aimed to examine dominant versus non-dominant limb performance using energy generation contribution of the hip, knee, and ankle during the vertical jump component of the lateral vertical jump. Isokinetic quadriceps strength and vertical jump height were not significantly different between limbs and each had small effect sizes. The dominant limb indicated greater hip energy generation contribution and lesser knee energy generation contribution when compared to the non-dominant limb, with no between-limb differences in ankle energy generation contribution.

We hypothesized female adolescent soccer players would produce greater total energy generation in the dominant 
Table 2: Dominant versus non-dominant limb comparisons.

\begin{tabular}{|c|c|c|c|c|c|c|}
\hline & \multicolumn{2}{|c|}{ Dominant } & \multicolumn{2}{|c|}{ Non-Dominant } & \multirow[b]{2}{*}{$p$-value } & \multirow[b]{2}{*}{ Cohen's d } \\
\hline & Mean & $S D$ & Mean & $S D$ & & \\
\hline Vertical jump height (m) & 0.12 & 0.03 & 0.13 & 0.03 & 0.59 & 0.33 \\
\hline Knee extension peak torque $60 \% \mathrm{~s}(\mathrm{Nm} / \mathrm{kg})$ & 1.79 & 0.51 & 1.82 & 0.45 & 0.64 & 0.06 \\
\hline Hip energy generation contribution (\%) & 29.7 & 8.5 & 18.4 & 6.3 & $<0.001$ & 1.51 \\
\hline Knee energy generation contribution (\%) & 22.8 & 6.8 & 36.2 & 8.5 & $<0.001$ & 1.74 \\
\hline Ankle energy generation contribution (\%) & 47.5 & 7.7 & 45.4 & 9 & 0.38 & 0.25 \\
\hline
\end{tabular}

$\mathrm{SD}=$ standard deviation

limb, indicated by greater vertical jump height. The lateral vertical jump adds frontal plane demand and increased task complexity, leading to our expectation of between-limb differences. However, vertical jump height was not significantly different between limbs. We also expected greater energy generation contribution in both the hip and knee on the dominant limb to comprise the hypothesized greater vertical jump height. Knee energy generation contribution was significantly lower on the dominant side, and there were no notable differences in vertical jump height between limbs. These findings did not align with our hypothesis.

In countermovement jump and drop jump tasks, performance is hip and knee dependent ${ }^{23}$ while the ankle has lesser contribution. ${ }^{24}$ These results are in contradiction with the findings of the current study in that the overall percentage of contribution of the ankle in both limbs (dominant $47.5 \pm 7.7 \%$, non-dominant $45.4 \pm 8.9 \%$ ) was higher than the contribution percentages of the hip and knee in either limb. While the current study focuses on between-limb differences rather than the percent of contribution from each individual joint, the fact that females relied on more of an "ankle strategy" during take-off does highlight task specific contributions of the ankle during the lateral vertical jump. Previous data demonstrates similar ankle contribution during the landing portion of the lateral vertical jump in healthy female adolescent athletes. ${ }^{22}$ While the current study was examining the percent contribution of each joint's ability to generate power, the former ${ }^{22}$ studied the ability to absorb forces or energy absorption contribution when landing from a jump. Nevertheless, young female athletes appear to rely on an ankle strategy during performance of a lateral vertical jump.

In the absence of observed power (vertical jump height) and strength (quadriceps strength) differences between limbs, energy generation contribution differences point to motor patterning. In general, hip, knee, and ankle muscle strength capacity are primary indicators of jump height, ${ }^{25}$ although motor control influences the extent with which that muscle capacity can be activated. ${ }^{26}$ Previous evidence indicates the role of dynamic stabilization and neuromuscular control in the landing phase of a single leg hop, with a balance training group demonstrating significantly reduced impact landing forces following training interventions. ${ }^{27}$ The motion in traditional countermovement jumps occur in the sagittal plane, aligned with the upcoming jump. In the lateral vertical jump, the preceding lateral hop phase adds a multiplanar component, necessitating frontal plane landing control. Neuromuscular control strategies from a lateral hop differ from landing in an anterior-posterior direction, requiring greater mechanical loading for lateral braking, ${ }^{28}$ and inducing greater demands on dynamic postural stability. ${ }^{29}$ As the end of the lateral hop phase of the lateral vertical jump occurs, frontal plane motion must be immediately transferred to the sagittal plane to perform the vertical jump. Jump performance is dependent on peak power development in the direction of the jump, ${ }^{30,31}$ For effective energy generation for the vertical jump phase, frontal plane deviations must be minimized. As such, the lateral vertical jump may be a good indicator of motor control as evidenced by energy generation contribution differences between limbs despite symmetrical jump heights.

Theorizing that the non-dominant limb better positions itself at the moment of energy transfer from the lateral hop into the vertical jump is feasible, highlighting the importance of coordination to perform the lateral vertical jump. Previous studies indicate the non-dominant limb functions in stability to allow the dominant limb to perform a manipulative task, 32 and the repetition of these unilateral actions may result in neuroplastic adaptations creating better proprioception and stabilization in the non-dominant limb. ${ }^{33}$ In soccer, the dominant limb functions to kick the ball while the non-dominant limb is necessary for stability. ${ }^{6}$ The nondominant limb in soccer players has demonstrated greater dynamic stability during single leg drop jumps as evidenced by knee valgus moment. ${ }^{34}$ It is reasonable that the nondominant limb performs this type of task more efficiently. On the contrary, with reversed roles, the dominant limb functioning as the landing "supporting" leg may have less neuromuscular control, ${ }^{34}$ driving a more hip-centric pattern to operate in the frontal plane to produce the vertical jump outcome. Perhaps clinicians should consider potential differences in strategies used to jump during this task in the current population. As such, interventions might include a greater focus on hip exercises in the non-dominant limb with more intention on knee or quadriceps exercises in the dominant limb.

\section{LIMITATIONS}

Generalizability of these results is limited to the cohort observed. These adolescent athletes are still developing, likely both in physical maturation and skill acquisition. It remains 
to be seen how more adult athletes, who might be more mature movers, might have different neuromuscular patterning and coordination. Differences in between-limb EGC may be attributed to coordination and motor control under the assumption that between-limb muscle strength is similar. Symmetry was observed in quadriceps muscle strength, but between-limb hip strength was not assessed to further examine this finding. The authors also assume that betweenlimb jump height can be attributed to power, strength, and coordination of the lower extremities. Other variables, such as intermuscular coordination using surface electromyography, ${ }^{35}$ or accounting for trunk and upper extremity positioning, may be useful in future studies.

\section{PRACTICAL APPLICATIONS}

Female adolescents in sport are a high-risk group for ACL injury, ${ }^{36}$ also demonstrating alarmingly high re-injury rates. ${ }^{37,38}$ Recent studies have reported the dominant limb in soccer players is more likely to sustain an ACL injury. ${ }^{39,40}$ Establishing outcomes for sport-specific tasks will help bridge the gap between traditional hop tests and full returnto-sport. Young females demonstrate movement patterns characterized by neuromuscular deficits ${ }^{41}$ that can be attributed to lacking strength and power, ${ }^{42}$ but also motor control and coordination. ${ }^{43}$ Continuing to study motor patterns in adolescent females may further elucidate the higher-level functional importance of the lateral vertical jump as a measure of dynamic stability. It is clear that different strategies for producing power are employed between the dominant and non-dominant limbs during the lateral vertical jump. Likewise, asymmetries in energy generation contribution at the hip and knee are apparent, but the clinical relevance of this finding remains difficult to ascertain. If the observable outcome (jump height) is symmetrical, but technique and patterning to achieve it (energy generation contribution) is asymmetrical, how should the task be classified?

For functional testing outcomes, attaining symmetry should be the standard. While arbitrary goals set at $90 \%$ of the contralateral limb ${ }^{44}$ or deeming a $15 \%$ difference as abnormal ${ }^{43}$ are common, meeting those cutoffs alone may not be sufficient. Grindem et al. ${ }^{45}$ reported that each percentage point closer to $100 \%$ symmetry in quadriceps strength resulted in a $3 \%$ decrease in re-injury rate in post-ACL reconstruction athletes. Functional tests should have some degree of sport-specificity if they intend to replicate onfield demands, and the lateral vertical jump appears applicable to numerous soccer-specific movement patterns. It is common for a soccer player to transition from a lateral movement into sagittal plane movement, all while negotiating space and potentially contacting an opponent. The present study sought to identify initial values for this novel task in vertical jump height. Independent of the aforementioned between-limb differences in EGC at the hip and knee, jump height was similar between limbs, indicating this cohort of healthy female adolescent soccer players is symmetrical in that regard. This preliminary data suggests the lateral vertical jump task can be used as an additional tool in rehabilitation and strength and conditioning, beyond traditional bilateral jumping tasks and unilateral but uniplanar tasks.

Symmetry normalization is a milestone in rehabilitation following injury but not the final goal. ${ }^{46}$ At some point, the intrinsic between-limb differences driven by inherent lateral dominance provide a ceiling. Even upon achieving symmetrical observable outcomes in between-limb vertical jump height, clinicians must recognize inherent "unseen" asymmetries and consider the intra-limb motor pattern alterations that may be used to achieve symmetry. While the results of the current study demonstrate similar power (vertical height) between limbs, the strategies used to achieve the outcome varied (i.e., greater hip, less knee energy generation contribution on the dominant limb versus less hip, greater knee energy generation contribution on the nondominant limb). The takeaways from the present study are that functional performance measures and striving for symmetry are not "black and white" - symmetry on the surface may not tell the underlying story. Symmetrical performance observed for jump height may be achieved via different underlying movement strategies.

\section{CONCLUSION}

The lateral vertical jump task is a novel measure of unilateral, multiplanar dynamic stability. The findings demonstrate interlimb symmetry in jump height during the lateral vertical jump task when comparing the dominant and nondominant limbs. However, different internal strategies were employed to achieve this outcome, demonstrated by higher knee energy generation contribution in the non-dominant limb and higher hip energy generation contribution in the dominant limb. Intra-limb symmetry must be considered within the context of the desired outcome, with the understanding that underlying joint-by-joint energy generation contribution differences may not coincide with overall outcomes.

\section{CONFLICTS OF INTEREST}

None declared.

\section{FUNDING}

None to report.

Submitted: July 06, 2020 CDT, Accepted: November 19, 2020 CDT 


\section{REFERENCES}

1. Fédération Internationale de Football Association. FIFA Women's Football Survey 2014. Fédération Internationale de Football Association; 2014.

2. Union of European Football Associations. Women's Football across the National Associations 2016/17. Union of European Football Associations; 2017.

3. Martínez-Lagunas V, Niessen M, Hartmann U. Women's football: Player characteristics and demands of the game. J Sport Health Sci. 2014;3(4):258-272. do i:10.1016/j.jshs.2014.10.001

4. Geertsema C. What are the differences between men's and women's football? Paper presented at the: XXVII Isokinetic Medical Group Conference; 2018.

5. Rouissi M, Chtara M, Owen A, et al. Effect of leg dominance on change of direction ability amongst young elite soccer players. J Sports Sci. 2016;34(6):542-548. doi:10.1080/02640414.2015.1129 432

6. DeLang MD, Rouissi M, Bragazzi NL, Chamari K, Salamh PA. Soccer Footedness and Between-Limbs Muscle Strength: Systematic Review and MetaAnalysis. Int J Sports Physiol Perform. 2019;14(5):551-562. doi:10.1123/ijspp.2018-0336

7. Nunome H, Lake M, Georgakis A, Stergioulas LK. Impact phase kinematics of instep kicking in soccer. J Sports Sci. 2006;24(1):11-22. doi:10.1080/0264041040 $\underline{0021450}$

8. Oliveira ASC, Barbieri FA, Gonçalves M. Flexibility, torque and kick performance in soccer: Effect of dominance. Sci Sports. 2013;28(3):e67-e70. doi:10.101 6/j.scispo.2013.01.004

9. Dörge HC, Andersen TB, Sørensen H, Simonsen EB. Biomechanical differences in soccer kicking with the preferred and the non-preferred leg. J Sports Sci. 2002;20(4):293-299.

10. Fältström A, Hägglund M, Kvist J. Functional Performance Among Active Female Soccer Players After Unilateral Primary Anterior Cruciate Ligament Reconstruction Compared With Knee-Healthy Controls. Am J Sports Med. 2017;45(2):377-385. doi:1 $\underline{0.1177 / 0363546516667266}$

11. Östenberg A, Roos E, Ekdah C, Roos H. Isokinetic knee extensor strength and functional performance in healthy female soccer players. Scand J Med Sci Sports. 1998;8(5):257-264.
12. Jones PA, Bampouras TM. A comparison of isokinetic and functional methods of assessing bilateral strength imbalance. J Strength Cond Res. 2010;24(6):1553-1558.

13. Reilly T. Energetics of high-intensity exercise (soccer) with particular reference to fatigue. J Sports Sci. 1997;15(3):257-263.

14. Mohr M, Krustrup P, Andersson H, Kirkendal D, Bangsbo J. Match activities of elite women soccer players at different performance levels. J Strength Cond Res. 2008;22(2):341-349.

15. Steffen K, Myklebust G, Olsen OE, Holme I, Bahr R. Preventing injuries in female youth football--a cluster-randomized controlled trial. Scand J Med Sci Sports. 2008;18(5):605-614. doi:10.1111/j.1600-083 8.2007.00703.x

16. Lindblom $H$, Waldén $M$, Hägglund $M$. No effect on performance tests from a neuromuscular warm-up programme in youth female football: a randomised controlled trial. Knee Surg Sports Traumatol Arthrosc. 2012;20(10):2116-2123. doi:10.1007/s00167-011-184 $\underline{6-9}$

17. Lockie RG, Moreno MR, Lazar A, et al. The physical and athletic performance characteristics of Division I collegiate female soccer players by position. J Strength Cond Res. 2018;32(2):334-343.

18. Oberacker LM, Davis SE, Haff GG, Witmer CA, Moir GL. The Yo-Yo IR2 test: physiological response, reliability, and application to elite soccer. J Strength Cond Res. 2012;26(10):2734-2740.

19. Ozbar N, Ates S, Agopyan A. The effect of 8-week plyometric training on leg power, jump and sprint performance in female soccer players. J Strength Cond Res. 2014;28(10):2888-2894.

20. Bishop C, Read P, McCubbine J, Turner A. Vertical and Horizontal Asymmetries are Related to Slower Sprinting and Jump Performance in Elite Youth Female Soccer Players. J Strength Cond Res. 2018;35(1):56-63. doi:10.1519/ISC.000000000000254 $\underline{4}$

21. Östenberg A, Roos H. Injury risk factors in female European football. A prospective study of 123 players during one season. Scand J Med Sci Sports. 2000;10(5):279-285. 
22. Boo ME, Garrison JC, Hannon JP, et al. Energy Absorption Contribution and Strength in Female Athletes at Return to Sport After Anterior Cruciate Ligament Reconstruction: Comparison With Healthy Controls. Orthop J Sports Med. 2018;6(3):2325967118759522. doi:10.1177/232596711 $\underline{8759522}$

23. von Elm E, Altman DG, Egger M, Pocock SJ, Gøtzsche PC, Vandenbroucke JP. The Strengthening the Reporting of Observational Studies in Epidemiology (STROBE) statement: Guidelines for reporting observational studies. Int J Surg. 2014;12(12):1495-1499. doi:10.1016/j.ijsu.2014.07.01 $\underline{3}$

24. Ford KR, Myer GD, Brent JL, Hewett TE. Hip and knee extensor moments predict vertical jump height in adolescent girls. J Strength Cond Res. 2009;23(4):1327-1331. doi:10.1519/jsc.0b013e31819b bea4

25. Vanezis A, Lees A. A biomechanical analysis of good and poor performers of the vertical jump. Ergonomics. 2005;48(11-14):1594-1603.

26. McErlain-Naylor S, King M, Pain MTG. Determinants of countermovement jump performance: A kinetic and kinematic analysis. $J$ Sports Sci. 2014;32(19):1805-1812. doi:10.1080/02640 $\underline{414.2014 .924055}$

27. Myer GD, Ford KR, Brent JL, et al. The effects of plyometric vs. dynamic stabilization and balance training on power, balance, and landing force in female athletes. J Strength Cond Res. 2006;20(2):345-353.

28. Silva PB, Oliveira AS, Mrachacz-Kersting N, Kersting UG. Effects of wobble board training on single-leg landing neuromechanics. Scand J Med Sci Sports. 2018;28(3):972-982. doi:10.1111/sms.13027

29. Wikstrom EA, Tillman MD, Schenker SM, Borsa PA. Jump-landing direction influences dynamic postural stability scores. J Sci Med Sport. 2008;11(2):106-111. doi:10.1016/j.jsams.2007.02.014

30. Murtagh CF, Vanrenterghem J, O’Boyle A, Morgans R, Drust B, Erskine RM. Unilateral jumps in different directions: A novel assessment of soccerassociated power? J Sci Med Sport. 2017;20(11):1018-1023. doi:10.1016/i.jsams.2017.03.0 $\underline{16}$

31. Fischer F, Blank C, Dünnwald T, et al. Isokinetic Extension Strength Is Associated With Single-Leg Vertical Jump Height. Orthop J Sports Med. 2017;5(11):232596711773676. doi:10.1177/232596711 $\underline{7736766}$
32. Grouios G, Hatzitaki V, Kollias N, Koidou I. Investigating the stabilising and mobilising features of footedness. Laterality. 2009;14(4):362-380. doi:10.1 080/13576500802434965

33. Sainburg RL. Evidence for a dynamic-dominance hypothesis of handedness. Exp Brain Res. 2002;142(2):241-258. doi:10.1007/s00221-001-0913-8

34. Ludwig O, Simon S, Piret J, Becker S, Marschall F. Differences in the dominant and non-dominant knee valgus angle in junior elite and amateur soccer players after unilateral landing. Sports. 2017;5(1):14. doi:10.3390/sports 5010014

35. van der Does HT, Brink MS, Benjaminse A, Visscher C, Lemmink KA. Jump Landing Characteristics Predict Lower Extremity Injuries in Indoor Team Sports. Int J Sports Med. 2016;37(3):251-256. doi:10.1055/s-0035-1559688

36. Prodromos CC, Han Y, Rogowski J, et al. A metaanalysis of the incidence of anterior cruciate ligament tears as a function of gender, sport, and a knee injury-reduction regimen. Arthroscopy. 2007;23(12):1320-1325.

37. Paterno MV, Rauh MJ, Schmitt LC, Ford KR, Hewett TE. Incidence of Second ACL Injuries 2 Years After Primary ACL Reconstruction and Return to Sport. Am J Sports Med. 2014;42(7):1567-1573. doi:1 $\underline{0.1177 / 0363546514530088}$

38. Webster KE, Feller JA. Exploring the High Reinjury Rate in Younger Patients Undergoing Anterior Cruciate Ligament Reconstruction. Am J Sports Med. 2016;44(11):2827-2832. doi:10.1177/03635465166518 $\underline{45}$

39. Della Villa F, Buckthorpe M, Grassi A, et al. Systematic video analysis of ACL injuries in professional male football (soccer): Injury mechanisms, situational patterns and biomechanics study on 134 consecutive cases. Br J Sports Med. 2020:2019-101247.

40. DeLang MD, Salamh PA, Farooq A, et al. The dominant leg is more likely to get injured in soccer players: Systematic review and meta-analysis. Biol Sport. 2021;38(3):397-405. doi:10.5114/biolsport.202 1.100265

41. Myer GD, Sugimoto D, Thomas S, Hewett TE. The influence of age on the effectiveness of neuromuscular training to reduce anterior cruciate ligament injury in female athletes: A meta-analysis. Am J Sports Med. 2013;41(1):203-215. doi:10.1177/036 $\underline{3546512460637}$ 
42. Myer GD, Ford KR, Palumbo JP, et al.

Neuromuscular training improves performance and lower-extremity biomechanics in female athletes. $J$ Strength Cond Res. 2005;19(1):51-60.

43. Hewett TE, Myer GD, Ford KR. Decrease in neuromuscular control about the knee with maturation in female athletes. J Bone Joint Surg Br. 2004;86(8):1601-1608. doi:10.2106/00004623-200408 000-00001

44. Croisier J-L, Ganteaume S, Binet J, Genty M, Ferret J-M. Strength imbalances and prevention of hamstring injury in professional soccer players: A prospective study. Am J Sports Med. 2008;36(8):1469-1475. doi:10.1177/036354650831676 $\underline{4}$
45. Grindem H, Snyder-Mackler L, Moksnes H, Engebretsen L, Risberg MA. Simple decision rules can reduce reinjury risk by $84 \%$ after ACL reconstruction: The Delaware-Oslo ACL cohort study. Br J Sports Med. 2016;50(13):804-808. doi:10.1136/bjsports-2016-0960 $\underline{31}$

46. Pairot de Fontenay B, Argaud S, Blache Y, Monteil K. Contralateral limb deficit seven months after ACLreconstruction: An analysis of single-leg hop tests. Knee. 2015;22(4):309-312. doi:10.1016/j.knee.2015.0 $\underline{4.012}$ 